\title{
Maintaining Consistency in Distributed Network Games
}

\author{
Kyung Seob Moon ${ }^{+} \quad$ Vallipuram Muthukkumarasamy ${ }^{+}$ \\ k.moon@griffith.edu.au v.muthu@griffith.edu.a \\ Anne Thuy-Anh Nguyen ${ }^{+}$ \\ Hyung Soo Kim* \\ a.nguyen@griffith.edu.au khs@hc.ac.kr \\ School of Information and Communication Technology, Griffith University \\ Gold Coast Campus, PMB 50, GCMC \\ Queensland 9726, Australia ${ }^{+}$ \\ Department of Computer Information Application, Cheju Halla College, Korea
}

\begin{abstract}
There are basically two approaches, conservative and optimistic, to maintain consistency in distributed network games. In the former, players may experience network latency, depending on packet transfer delay, caused by the send-and-wait frame and acknowledgement packets. In the latter approaches, the processes do not wait for other players' packets and advance to their own frames, hence no network latency. However, when inconsistency happens the processes must roll back. These can cause irritation and confusion to players. Overall, the optimistic approaches may not be suitable for networked games. To overcome the network latency problem in the conservative approaches, this paper introduces a network system which can reduce network latency and bandwidth by utilizing packet-relay and shortest path algorithms. Furthermore, game efficiency comparison between lock-step and bucket-synchronization algorithms on the proposed network system is presented in this paper.
\end{abstract}

Keywords- consistency maintenance, distributed games, low-latency, peer-to-peer network.

\section{INTRODUCTION}

Multiplayer Online Games (MOG) prefer Client/Server (C/S) network architecture to Peer-to-Peer (P2P) because of its advantages such as simplicity of consistency maintenance, better security, authentication, and easy billing system. However, C/S architecture can cause network latency and server(s) can become network bottleneck [1]. To solve the bottleneck problem, server clustering methods can be used, however these may not be cost effective.

Other types of games use P2P network architecture but the total number of players in one game session is limited because of network bandwidth problems. In addition, consistency maintenance problems must be solved in $\mathrm{P} 2 \mathrm{P}$ architecture.
In $\mathrm{C} / \mathrm{S}$ structure, clients send packets which consist of object states or user commands to the server. Subsequently the server determines the validity of the packets and sends back the result to the client. The states of all objects in the game are kept in the server side, so there is no or less inconsistency problem compared with the P2P structure. On the other hand, each node manages its own object states in the P2P structure so, inconsistency can occur in case of network delay.

As mentioned above, $\mathrm{C} / \mathrm{S}$ structure can cause network latency because of the two-way packet transfer which is from Client to Server and then from Server to Client again. So, if $T$ is the network latency between Server and Client then $2 \mathrm{~T}$ is required for communication. However, only $1 \mathrm{~T}$ is necessary in $\mathrm{P} 2 \mathrm{P}$ structure because movement validations are made in each peer.

If P2P uses unicast and unstructured overlay then each node sends the same packets to all other peers and this will cause network bandwidth problems. This paper examines tree-based P2P network system which helps to resolve the P2P network latency and bandwidth problems.

In the next section, related work in consistency maintenance area is briefly reviewed. In section 3, basic architecture of Tree-Based P2P system is introduced. Experiment related facts and result are shown in section 4. Finally, conclusions and future work are presented in section 5 .

\section{RELATED WORK}

The Lock-Step algorithm [2] is one of the simplest solutions for consistency maintenance in the P2P structure. Each peer waits for other peers' packets of current frame, makes its next move, sends packets and waits again. The drawback of this approach is that it can cause slowdown for game play if network latency is slower than the frame rate. For example, if the game's FPS (Frame per Second) is 25 then each frame takes about $40 \mathrm{~ms}$ 
to load. In case the network latency is longer than $40 \mathrm{~ms}$ then players will have to wait until they get other players' packets.

Frequent State Regeneration [8] approach eliminates the slowdown-time of the lock-step algorithm by frequently transmitting the status of objects in game sessions. Generally, an unreliable protocol such as UDP is used with this approach to alleviate the overhead of using a reliable protocol such as TCP. However, sending the status of objects frequently to all players requires high bandwidth and this requirement limits the maximum number of players for network games.

Bucket Synchronization algorithm [5] and Local Lag [3] introduce artificial delay so as to synchronize its own frame with other nodes' frame by utilizing imperfect human visual perception ability. This approach is the analogous to the buffering method of streaming audio. Even though the lock-step time is extended, it still requires inconsistency resolution algorithms because network latency is longer than the extended lock-step time. The approaches used in [3] and [5] also require high bandwidth due to frequent state transmission they had adopted in their solutions.

Dead Reckoning [4] algorithms interpolate and/or extrapolate missing and/or incoming information to reduce bandwidth requirement and latency. Local Perception Filter [9] approach also utilizes the limitation of human eye perception by altering the speed of objects in networking games for hiding network latency. Time Warp [6] algorithm has been introduced to solve inconsistency and/or network latency problems by adapting optimistic approaches. However, the overhead of rollover process is unavoidable.

\section{TREE-BASED P2P NETWORK SYSTEM}

To maintain fair games for all players in a game session, the game speed is synchronized with the slowest process and network latency node. There are two variables: process speed and network latency. The first variable, process speed, is easy to test before game sessions start and it is not changed dramatically during game sessions. Therefore, process speed problem can be solved by a pre-test to ascertain whether players' computers can process adequate frame rate during game sessions.

However, network latency is hard to be predicted correctly and it is also not consistent during game sessions. Therefore, a tree-based P2P system is proposed and explained in the following sections.

\section{A Graph to tree conversion}

Existing P2P games use a graph structure to format network topology in which each node connects to every other nodes. As explained before, this can cause bandwidth problem if unicast is used. Also, all nodes are synchronized with the slowest node, so this structure cannot take advantage of fast connections between other nodes.
On the other hand, if network topology is structured by tree-based architecture then nodes will be hierarchical and fast connection nodes will be grouped together. Figure 1 shows the graph structure and network latency values between nodes.

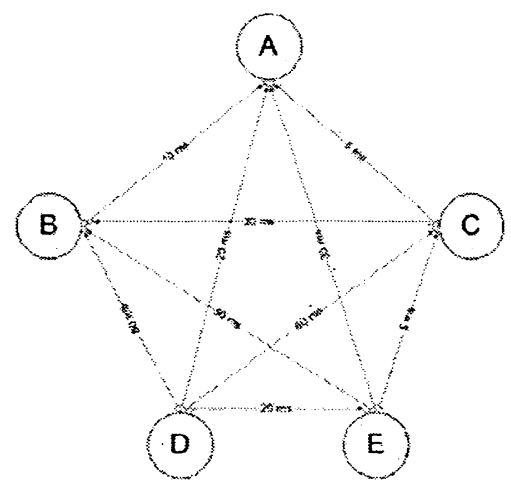

Fig. 1. Graph structure for network topology and network latency values between nodes.

Figure 2 displays the final result after removing high network latency links from the graph structure. As shown in figure 3, node A creates its own tree structure from the graph structure.
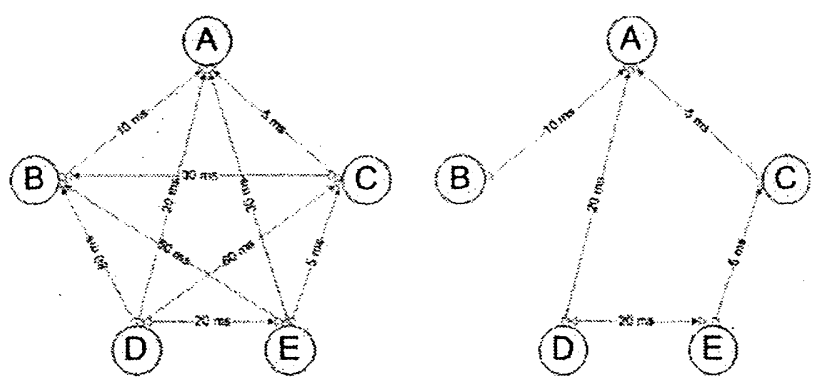

Fig. 2. Finding alternative paths for high network latency nodes.

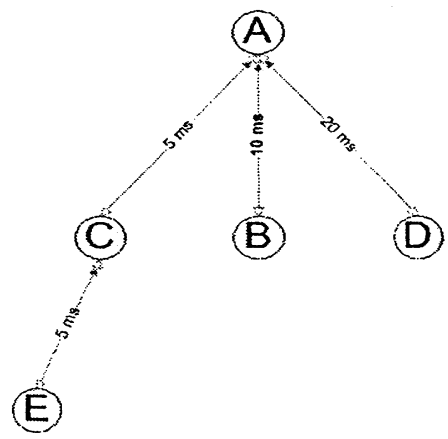

Fig. 3. Conversion from graph structure to tree structure for node A.

As it can be seen in figure 1, the longest path from node $A$ to other nodes is the link between A and D which takes $20 \mathrm{~ms}$. Therefore, we create the $\mathrm{A}-\mathrm{C}-\mathrm{E}$ path because the total time from node $A$ to $E$ is $10 \mathrm{~ms}$, which is shorter than $20 \mathrm{~ms}$ ( $A$ to $D$ ). 
Furthermore, the new path reduces network latency by $20 \mathrm{~ms}$ since the latency from $\mathrm{A}$ to $\mathrm{E}$ is $30 \mathrm{~ms}$ if direct link is used.

The most important aspect is that this conversion can increase the overall game speed by reducing the network latency of the slowest link in the network topology. Again, as it can be seen from figure 1, the B-D connection is the slowest link and the B-A-D link can reduce network latency by $50 \mathrm{~ms}$. For graph to tree conversion, one of the most well known shortest path finding algorithm, Dijkstra algorithm [7], is used.

\section{B. Relay Method}

Nodes in this tree-based P2P system may need to relay packets to its child nodes because the sender nodes may not have direct connection to destination nodes. To create the relay process, the sender node becomes a root node and transmits packets to its own child nodes which are one level below nodes only. The sender node includes the identification numbers of destination nodes in its packet header and when its child nodes receive the packet they remove their id from the packet header and relay this packet to their own child nodes.

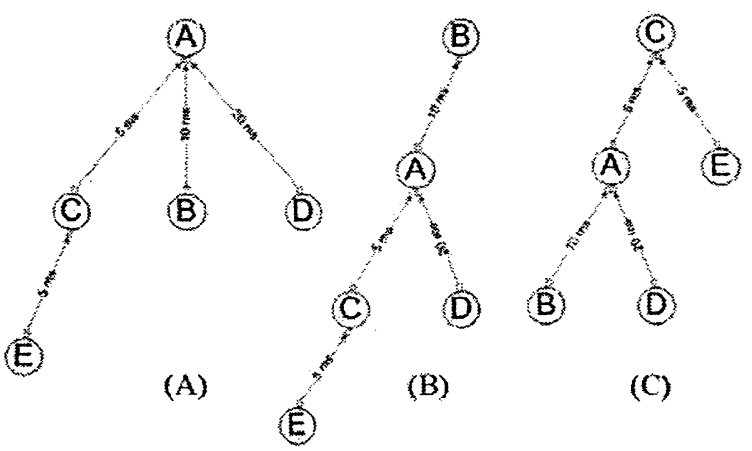

Fig. 4. Tree Structures for each node (Node A, B, and C only)

Figure 4 shows the tree structures for three nodes. When node A sends a packet to other nodes, it includes the ids of destination nodes. To send a packet from $\mathrm{A}$ to $\mathrm{E}$, the destination node list will be $[C, E]$. Because node $B$ and $D$ do not have child nodes, their destination node lists will be [B] and [D] respectively. When node $C$ receives the packet from node $A$, it removes itself from the destination node list and relays the packet to node $\mathrm{E}$ only (because node $\mathrm{A}$ is not in the destination node list). The following pseudo codes show the relay algorithm in detail.

\section{Retransmission}

Retransmission is unavoidable when packet drop or corruption happens, especially when frequent packet regeneration scheme [8] is not used. The decision for retransmissions is based on $\mathrm{ACK}$ timeout and $\mathrm{ACK}$ numbers from opponent nodes. The retransmission procedure is exactly the same as sending packets at the first time except the retransmission will occur at the parent node of the node that requests the missing packets.

\section{Acknowledgement}

This tree based P2P system can prevent ACK implosion problems because, each node manages its own child/ children. When a packet is sent, the destination node sends ACK to its origin node immediately. The sender retransmits the packet if (ACK_timeout_of_destination node Current_Waiting_Time_For_ACK) is true. The ACK timeout is based on ping time between the two nodes.

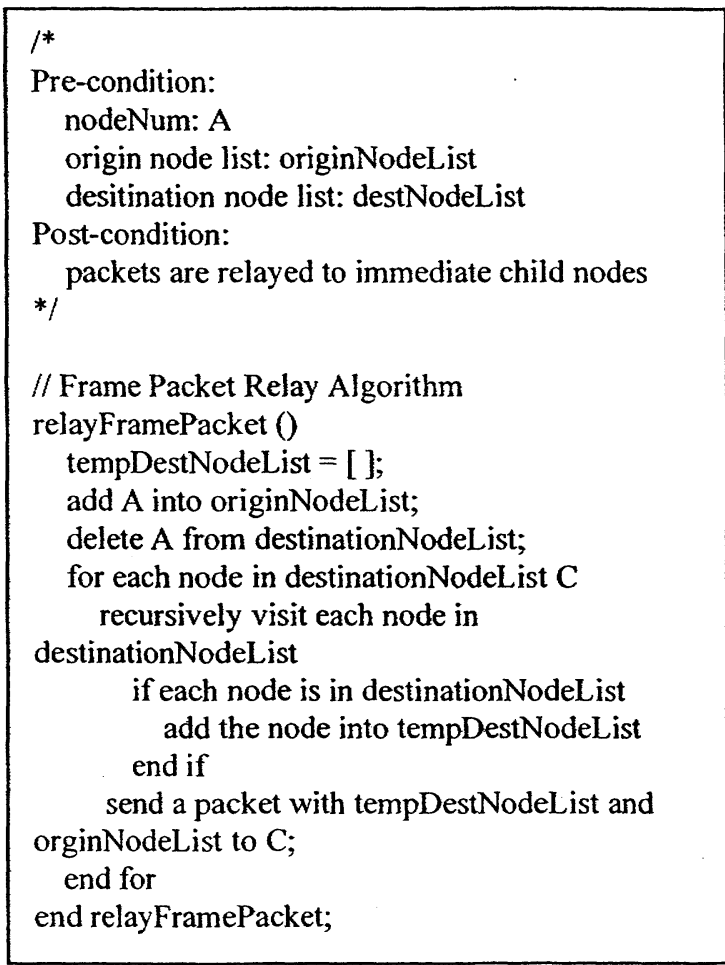

\section{E. Simulator Architecture}

The tree-based P2P network simulator is implemented in $\mathrm{C}++$ with STL and consists of three main classes, namely, Player, Simulator and Statistics. The player class utilizes three data structure types, Queue for an input buffer, Priority Queue for a re-sending buffer, and Circular Array for a game buffer to store other players' packets, to re-send dropped packets, and to gather frame data respectively. Figure 5 shows the basic architecture of the simulator. 


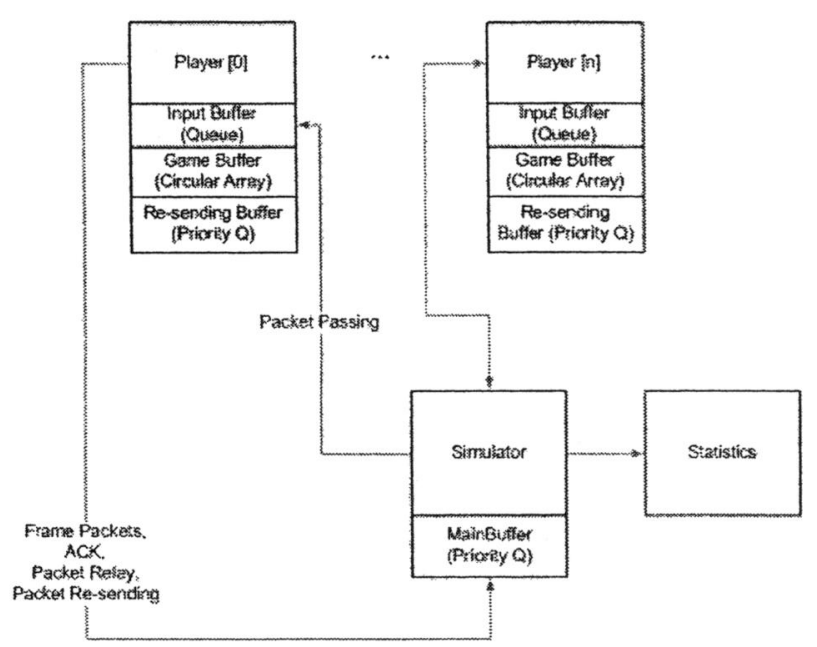

Fig. 5. Tree-based P2P Network Simulator Architecture

\section{PRELIMINARY EVALUATION}

The tree-based and graph-based P2P networks are evaluated and their performances are compared in this section. An Intel Pentium $4 \mathrm{CPU} 1.60 \mathrm{GHz}$ equipped computer with Microsoft windows XP operating system and 512MB RAM was used for experiments.

\section{A. Experimental Data Set}

The simulator initially generated a data set for eight players with randomly generated network latency between $5 \mathrm{~ms}$ and $100 \mathrm{~ms}$.

TABLE 1

LATENCY VALUES (ms) BETWEEN PLAYERS

\begin{tabular}{|c|c|c|c|c|c|c|c|c|}
\hline & 0 & 1 & 2 & 3 & 4 & 5 & 6 & 7 \\
\hline 0 & 0 & 26 & 58 & 76 & 10 & 46 & 11 & 60 \\
\hline 1 & 26 & 0 & 64 & 52 & 37 & 58 & 36 & 28 \\
\hline 2 & 58 & 64 & 0 & 15 & 48 & 77 & 35 & 54 \\
\hline 3 & 76 & 52 & 15 & 0 & 18 & 15 & 21 & 24 \\
\hline 4 & 10 & 37 & 8 & 18 & 0 & 27 & 77 & 25 \\
\hline 5 & 46 & 58 & 77 & 15 & 27 & 0 & 76 & 9 \\
\hline 6 & 11 & 36 & 35 & 21 & 77 & 76 & 0 & 70 \\
\hline 7 & 60 & 28 & 54 & 24 & 25 & 9 & 70 & 0 \\
\hline Sum: & 287 & 301 & 311 & 221 & 242 & 308 & 326 & 270 \\
\hline Avg: & 41 & 43 & 44.43 & 31.57 & 34.57 & 44 & 46.57 & 38.57 \\
\hline
\end{tabular}

\section{B. Comparison between graph-based and tree-based} structure

Frame rate and average latency were recorded on four different occasions, which were created using two consistency maintenance algorithms (bucket synchronization and lockstep) and two network types namely, graph-based, and tree-based.
Figure 6 and Table 2 show the changes of frame rate according to drop rate in the four occasions. The frame rate dropped to 24.78 and 23.9 from 25 when drop rate was $5 \%$ and $10 \%$ respectively for a bucket and tree combination. However, for bucket and graph combination Table 2 shows that the frame rates became 20.3 and 18.13 with $5 \%$ and $10 \%$ drop rate respectively.

TABLE 2

FRAME RATE (FPS) vs. DROP RATE(\%)

\begin{tabular}{ccccc}
\hline \hline Drop Rate & Bucket + Tree & $\begin{array}{c}\text { Bucket }+ \\
\text { Graph }\end{array}$ & $\begin{array}{c}\text { Lockstep }+ \\
\text { Tree }\end{array}$ & $\begin{array}{c}\text { Lockstep + } \\
\text { Graph }\end{array}$ \\
\hline 0 & 25 & 25 & 12.52 & 12.51 \\
1 & 25 & 23.62 & 12.08 & 9.32 \\
2 & 25 & 22.37 & 11.57 & 8.38 \\
5 & 24.78 & 20.3 & 10.77 & 7.07 \\
10 & 23.9 & 18.13 & 9.42 & 5.95 \\
\hline \hline
\end{tabular}

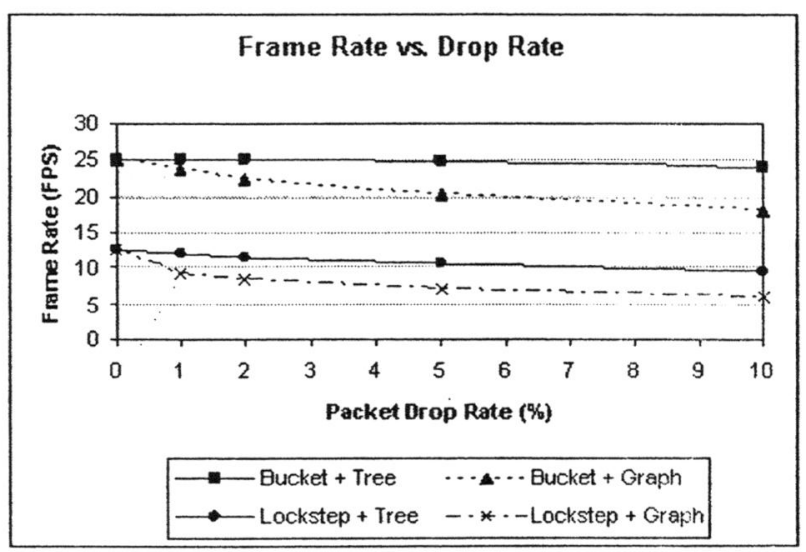

Fig. 6. Frame Rate vs. Drop Rate

The graphs of the average packet propagation time on packet drop rate of the four combinations are shown in figure 7. As it can be seen in figure 7, the latency of the two graph-based approaches is similar and so is the latency of the two tree-based approaches. According to the table 3, the graph to tree conversion improved the network delay from 39.74 io 26.93 when the packet drop rate was $0 \%$ and the change rate was linear. 
TABLE 3

LATENCY (ms) vs. DROP RATE (\%)

\begin{tabular}{ccccc}
\hline \hline Drop Rate & $\begin{array}{c}\text { Bucket }+ \\
\text { Tree }\end{array}$ & $\begin{array}{c}\text { Bucket }+ \\
\text { Graph }\end{array}$ & $\begin{array}{c}\text { Lockstep + } \\
\text { Tree }\end{array}$ & $\begin{array}{c}\text { Lockstep }+ \\
\text { Graph }\end{array}$ \\
\hline 0 & 26.93 & 39.74 & 26.93 & 39.74 \\
1 & 27.46 & 40.49 & 27.45 & 40.58 \\
2 & 27.99 & 41.33 & 28.07 & 41.39 \\
5 & 29.59 & 43.85 & 29.44 & 43.63 \\
10 & 32.51 & 48.35 & 32.54 & 47.86 \\
\hline \hline
\end{tabular}

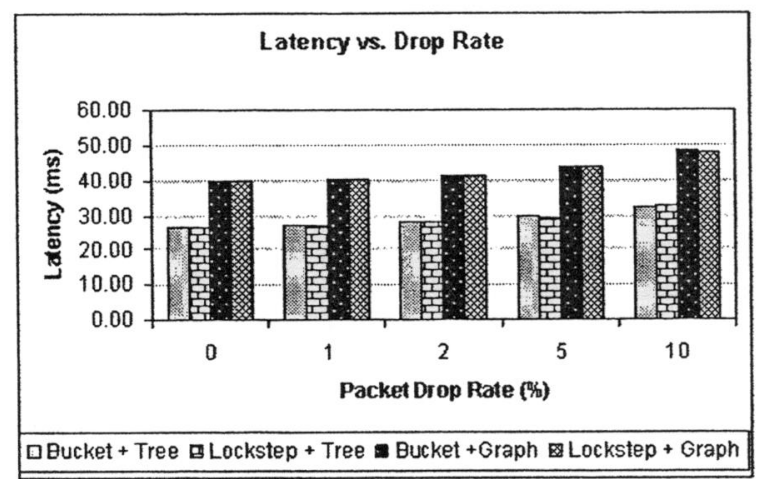

Fig. 7. Latency vs. Drop Rate

Figure 8 , Table 4 , and Table 5 show the maximum frame latency in each frame on two cases: graph-based and tree-based P2P networks. For both cases, the bucket synchronization algorithm is used. The average delayed frame rates for graph-based and tree-based approaches are $14.58 \%$ and $1.58 \%$ respectively.

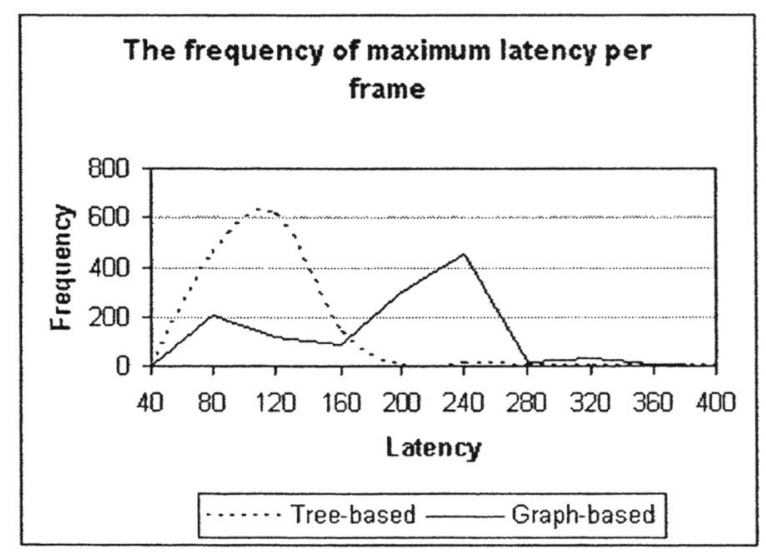

Fig. 8. Max latency of each frame in the two approaches ( $5 \%$ drop rate, 120 ms playout delay and $40 \mathrm{~ms}$ frame interval).
TABLE 4

FREQUECY OF DELAYED FRAME (GRAPH-BASED)

\begin{tabular}{cccc}
\hline \hline Player & No. Frames & Delayed Frames & Delay Rate \% \\
\hline 0 & 1215 & 235 & 19.34 \\
1 & 1217 & 174 & 14.3 \\
2 & 1215 & 224 & 18.44 \\
3 & 1215 & 124 & 10.21 \\
4 & 1215 & 64 & 5.27 \\
5 & 1214 & 232 & 19.11 \\
6 & 1214 & 178 & 14.66 \\
7 & 1214 & 186 & 15.32 \\
\hline \hline
\end{tabular}

Consistency algorithm: Bucket-synchronization

Network type: Graph

Frame interval: $40 \mathrm{~ms}$

Average delay rate: $14.58 \%$

Packet drop rate: $5 \%$

Playout delay: $120 \mathrm{~ms}$

TABLE 5

FREQUECY OF DELAYED FRAME (TREE-BASED)

\begin{tabular}{cccc}
\hline \hline Player & No. Frames & Delayed Frames & Delay Rate \% \\
\hline 0 & 1485 & 0 & 0 \\
1 & 1482 & 99 & 6.68 \\
2 & 1483 & 4 & 0.27 \\
3 & 1482 & 76 & 5.13 \\
4 & 1483 & 2 & 0.13 \\
5 & 1484 & 3 & 0.2 \\
6 & 1485 & 2 & 0.13 \\
7 & 1483 & 1 & 0.07 \\
\hline \hline
\end{tabular}

Consistency algorithm: Bucket-synchronization

Network type: Tree $\quad$ Packet drop rate: 5\%

Frame interval: $40 \mathrm{~ms}$

Playout delay: $120 \mathrm{~ms}$

Average delay rate: $1.58 \%$

\section{CONCLUSION}

Network latency and bandwidth are important factors to the playability of network games. Packet drop rate is the most important factor which can cause network latency. In this paper, we investigated the effects of packet drop rate on two P2P approaches. We have evaluated the efficiency of the tree-based P2P approach by comparing its performances with that of the graph-based P2P approach. Two consistency maintenance algorithms, lockstep and bucket synchronization, were implemented to analyze the efficiency of the tree-based approach. Our experimental results showed that the tree-based approach with the bucket synchronization algorithm performed close to optimal frame rate, even with a $10 \%$ packet drop rate.

Therefore, the tree-based peer-to-peer network system with bucket synchronization algorithm is more appropriate for the Internet games, which suffer mainly from unpredictable network latency and packet drops. For further performance enhancements, the effect of the tree-based approach on network bandwidth under dynamic drop rate conditions will be investigated in future. 


\section{REFERENCES}

[1] N. Baughman, and B. Levine, "Cheat-proof playout for centralized and distributed online games," In Proc. Infocom 2001, April 2001. Wadsworth, 1993, pp. 123-135.

[2] O. Wolfson, "The overhead of locking (and commit) protocols in distributed databases," ACM Transactions on Database Systems (TODS), v. 12 n.3, p. 453-471, Sept. 1987

[3] G. Laurent, and D. Christophe, "Design and evaluation of MiMaze a multi-player game on the Internet," In Proceedings of Multimedia Computing and Systems IEEE International Conference, p233-236, 28 June-1 July 1998.

[4] S. Singhal, "Effective remote modelling in large-scale distributed simulation and visualization environments," $\mathrm{PhD}$ dissertation. Department of Computer Science, Stanford University, Palo Alto, August 1996.

[5] J. Vogel, and M. Martin, "Network Games: Consistency control for distributed interactive media," In Proceedings of the ninth ACM international conference on Multimedja, October 2001.

[6] D. R. Jefferson, "Virtual Time," ACM Transactions on Programming Languages and Systems, 7(3):404-425, July 1985.

[7] E. W. Dijkstra, "A note on two problems in connexion with graphs," In: Numerische Mathematik 1 (1959), S. 269-271.

[8] S. Singhal, and Z. Michael, "Networked Virtual Environments: Design and Implementation," Addison Wesley, ACM Press, July 1999.

[9] M.S. Paul, D.R. Matthew, and J.R. David, “A Local Perception Filter for Distributed Virtual Environments," IEEE Virtual Reality Annual International Symposium (VRAIS 98), Atlanta, GA, 14-16 Mar., 1998. 